\title{
CONSTRUCTIVE PROOF OF THE EXISTENCE OF MULTIPLICATIVE FUNCTIONALS IN COMMU- TATIVE SEPARABLE BANACH ALGEBRAS
}

\author{
BY H. G. GARNIR, M. DE WILDE AND J. SCHMETS
}

Communicated by H. Helson, Feb. 15, 1967

Gelfand's 1941 proof of the existence of multiplicative functionals in commutative Banach algebras is essentially based on Zorn's axiom.

In 1961, P. J. Cohen [3] gave a constructive (i.e. free from Zorn's axiom) way to get rid of Banach algebras in some of their applications.

This year, E. Bishop [1], [2] has presented a theory of Banach algebras in the frame of L. E. J. Brouwer's constructivist ideas. Therefrom it is easy to deduce a constructive proof of the existence of multiplicative functionals. However this proof would be needlessly intricate when just interested in constructive methods.

Here is a simple constructive proof of Gelfand's theorem.

1. Let $A$ be a commutative separable Banach algebra with unit 1 throughout the paper.

Let us recall some properties of ideals of $A$.

(a) $0, A, \sum_{i=1}^{m} x_{i} A$ and $J+\sum_{i=1}^{m} x_{i} A$ are ideals of $A$ whenever $x_{1}, \cdots, x_{m} \in A$ and $J$ is an ideal of $A$.

(b) If an ideal $\Im$ contains an invertible element, then $I=A$.

(c) Let $\Im \neq A$ be an ideal, then $d[1, \Im]=1$.

Since $0 \in J, d[1, J] \leqq 1$. Moreover if $d[1, J]<1$, there exists $x_{0} \in J$ such that $d\left[1, x_{0}\right]<1$. Then $x_{0}^{-1}$ exists and consequently $1=x_{0} x_{0}^{-1}$ belongs to $\mathfrak{J}$.

(d) Let $\Im \neq A$ be an ideal. If $1-x y \in \Im$, then $d[x, \Im] \geqq 1 /\|y\|$.

In fact, $\Im \neq A$ implies $d[1, J]=1$ and since $1-x y \in J$, we have $d[x y, \jmath]=1$ and $d[x y, \jmath] \leqq d[x y, y \jmath] \leqq\|y\| d[x, \Im]$.

2. We need a lemma, which is a direct version of the classical fact that the spectrum of the Banach algebra $E / A$ is not void.

Let $\exists \neq A$ be an ideal. Then for all $x \in A$, there exists $z \in C$ such that $3+(x-z) A \neq A$.

Suppose there exists an ideal $\Im \neq A$ and $x \in A$ such that $\Im+(x-z) A$ $=A$ for all $z \in C$.

Then for all $z \in C$, there is at least one element $a(z) \in A$ with $1-(x-z) a(z) \in J$.

Let $\mathfrak{x}$ be any continuous linear functional in $A$ vanishing on $\mathfrak{J}$.

(a) $\mathfrak{x}[a(z)]$ depends only on $z \in C$ and not on the choice of $a(z)$. 
In fact if $1-(x-z) a_{i} \in J,(i=1,2)$, then

$$
\mathfrak{x}\left[a_{1}-a_{2}\right]=\mathfrak{x}\left[\left(1-(x-z) a_{2}\right) a_{1}-\left(1-(x-z) a_{1}\right) a_{2}\right]=0 .
$$

So $\mathfrak{x}[a(z)]$ is defined without using the axiom of choice.

(b) $\mathfrak{x}[a(z)]$ is holomorphic on $\boldsymbol{C}$.

In the neighborhood $V\left(z_{0}\right)=\left\{z:\left|z-z_{0}\right|<1 /\left\|a\left(z_{0}\right)\right\|\right\}$ of $z_{0} \in C$, $1-\left(z-z_{0}\right) a\left(z_{0}\right)$ is invertible and $\mathfrak{x}[a(z)]=\mathfrak{x}\left[a\left(z_{0}\right) /\left(1-\left(z-z_{0}\right) a\left(z_{0}\right)\right)\right]$ since

$$
\begin{aligned}
& a(z)-\frac{a\left(z_{0}\right)}{1-\left(z-z_{0}\right) a\left(z_{0}\right)} \\
& \quad=\frac{\left[1-\left(x-z_{0}\right) a\left(z_{0}\right)\right] a(z)-[1-(x-z) a(z)] a\left(z_{0}\right)}{1-\left(z-z_{0}\right) a\left(z_{0}\right)} \in J .
\end{aligned}
$$

(c) In fact, $\mathfrak{x}[a(z)] \equiv 0$.

For $|z|>\|x\|, x-z$ is invertible and $\mathfrak{x}[a(z)]=\mathfrak{x}\left[(x-z)^{-1}\right]$ because

$$
(x-z)^{-1}-a(z)=(x-z)^{-1}[1-(x-z) a(z)] \in J .
$$

Hence the conclusion by Liouville's theorem, since $\mathfrak{x}\left[(x-z)^{-1}\right] \rightarrow 0$ when $z \rightarrow \infty$, from the inequalities

$$
\left|\mathfrak{x}\left[(x-z)^{-1}\right]\right| \leqq C\left\|(x-z)^{-1}\right\| \leqq C(|z|-\|x\|)^{-1} .
$$

(d) There exists $\mathfrak{x}$ vanishing on $\mathfrak{J}$ and such that $\mathfrak{x}[a(z)] \not \equiv 0$.

Let us fix $z_{0} \in C$. Since $d[1, J]=1$ and $1-\left(x-z_{0}\right) a\left(z_{0}\right) \in J$, we have $d\left[a\left(z_{0}\right), \Im\right] \geqq 1 /\left(\|x\|+\left|z_{0}\right|\right)$. As $A$ is separable, by Hahn-Banach's theorem (see [4], for instance), there is a continuous linear functional x such that

$$
\mathfrak{x}\left[a\left(z_{0}\right)\right]=1 /\left(\|x\|+\left|z_{0}\right|\right), \quad \mathfrak{x}[\mathfrak{J}]=0 .
$$

So (c) and (d) are contradictory, hence the lemma.

3. Let us prove Gelfand's theorem.

Let $A$ be a commutative separable Banach algebra with unit 1.

If $J \neq A$ is an ideal, then there exists a continuous nonzero multiplicative functional vanishing on $J$.

Let $x_{m}$ be a dense sequence in $A$.

Since $J \neq A$, by successive applications of the lemma we get a sequence $z_{m} \in C$ such that $I+\sum_{i=1}^{m}\left(x_{i}-z_{i}\right) A \neq A$, hence

$$
d\left[1, \mathfrak{J}+\sum_{i=1}^{m}\left(x_{i}-z_{i}\right) A\right]=1, \quad \forall m .
$$


Therefore there exists a sequence $\mathfrak{x}_{m}$ of continuous linear functionals such that

$$
\left\|\mathfrak{x}_{m}\right\|=1, \quad \mathfrak{x}_{m}(1)=1, \quad \mathfrak{x}_{m}\left[\mathfrak{J}+\sum_{i=1}^{m}\left(x_{i}-z_{i}\right) A\right]=0 .
$$

Hence we get

for

$$
\begin{aligned}
\mathfrak{x}_{m}(\mathfrak{J}) & =0, \mathfrak{x}_{m}\left(x_{i}\right)=z_{i}, \\
\mathfrak{x}_{m}\left(x_{i} x_{j}\right) & =z_{i} \mathfrak{x}_{m}\left(x_{j}\right)=\mathfrak{x}_{m}\left(x_{i}\right) \mathfrak{x}_{m}\left(x_{j}\right), \quad \forall i, j \leqq m
\end{aligned}
$$

$$
J, x_{i}-z_{i}, x_{i} x_{j}-z_{i} x_{j} \in \mathfrak{J}+\sum_{i=1}^{m}\left(x_{i}-z_{i}\right) A .
$$

As $\left\|\mathfrak{x}_{m}\right\|=1$ for all $m$, there is a weak convergent subsequence of $\mathfrak{x}_{m}$ for $A$ is separable (see [4], for instance). Let $\mathfrak{x}$ be its limit.

Of course $\mathfrak{x}$ is a continuous linear functional and

$$
\|\mathfrak{x}\|=1, \quad \mathfrak{x}(1)=1, \quad \mathfrak{x}(J)=0 .
$$

Moreover $\mathfrak{x}$ is a multiplicative functional. In fact, we have

$$
\mathfrak{x}\left(x_{i} x_{j}\right)=\mathfrak{x}\left(x_{i}\right) \mathfrak{x}\left(x_{j}\right), \quad \forall i, j
$$

and the sequence $x_{m}$ is dense in $A$.

\section{REFERENCES}

1. E. Bishop, Seminar in La Jolla, 1965-1966.

2. - On constructivist methods in analysis, Chapter XI, Abstracts of Reports of the International Congress of Mathematics, Moscow, 1966, p. 39.

3. P. J. Cohen, A note on constructive methods in Banach algebras, Proc. Amer. Math. Soc. 12 (1961), 159-163.

4. H. G. Garnir, M. De Wilde, J. Schmets, Thêorie constructive des espaces lineaires a semi-normes, Sém. An. Math. et Alg. Univ. Liège, 1962-1966. University of Liège, Belgium.

UNIVERSiTy OF Lì̀GE, BelgiUM 